\title{
Community-acquired infections due to Staphylococcus argenteus lineage isolates harbouring the Panton- Valentine leucocidin, France, 2014
}

C Dupieux (celine.dupieux@chu-lyon.fr) ${ }^{1,2}$, R Blondé $^{3}$, C Bouchiat $^{1,2}$, H Meugnier $^{1,2}$, M Bes $^{1,2}$, S Laurent ${ }^{3}$, F Vandenesch $^{1,2}$, F Laurent ${ }^{1,2}$, A Tristan ${ }^{1,2}$

1. Centre National de Référence des Staphylocoques, Centre de Biologie et de Pathologie Est, Hospices Civils de Lyon, Bron, France

2. CIRI, International Center for Infectiology Research, INSERM U1111, CNRS UMR5308, Université de Lyon, Ecole Normale Supérieure de Lyon, France

3. Service de Réanimation, Centre Hospitalier de Mayotte, Mamoudzou, France

Citation style for this article:

Dupieux C, Blondé R, Bouchiat C, Meugnier H, Bes M, Laurent S, Vandenesch F, Laurent F, Tristan A. Community-acquired infections due to Staphylococcus argenteus lineage isolates harbouring the Panton-Valentine leucocidin, France, 2014. Euro Surveill. 2015;20(23):pii=21154. Available online: http://www. eurosurveillance.org

We describe two cases of human infections caused by Staphylococcus aureus clonal complex (CC) 75 , also called Staphylococcus argenteus, harbouring the Panton-Valentine leucocidin (PVL). These two sporadic cases were community-acquired, and identified in France in 2014. Both had an epidemiological link with Mayotte, an overseas department of France located in the Indian Ocean off the south-eastern African coast. This report illustrates that, contrary to previous descriptions, $S$. argenteus can acquire important virulence factors and be responsible for severe infections.

In 2014, two cases of human infections caused by Staphylococcus aureus isolates belonging to clonal complex (CC) 75 lineage, also called Staphylococcus argenteus, harbouring the Panton-Valentine leucocidin (PVL) occurred in France. The two cases were sporadic and community-acquired, and had an epidemiological link with Mayotte, a French archipelago in the Indian Ocean. The two strains affecting the respective cases were both isolated from blood culture.

\section{Description}

\section{Case 1}

In January 2014, a French woman of Comorian origin in her mid-twenties presented to the Emergency Department of a hospital in Lyon, with an eight-day history of deterioration of the general condition, chills and cough complicated by haemoptysis starting the day before. The symptoms had appeared three weeks after the excision of a recurrent abscess in the left thigh for which the patient had initially received oral treatment with amoxicillin-clavulanic acid during five days, then roxithromycin during the following eight days. Interview of the patient revealed a travel to Mayotte six months earlier. The patient was immediately admitted to hospital. At that time, she presented with cough, haemoptysis and chest pain. Body temperature was high at $39.1^{\circ} \mathrm{C}$ and heart rate was $119 / \mathrm{min}$. Laboratory findings showed a white blood cell count of $17.4 \times 10^{9} / \mathrm{L}$ (norm: $4-10.5 \times 10^{9} / \mathrm{L}$ ), a C-reactive protein (CRP) of $251 \mathrm{mg} / \mathrm{L}$ (norm: $<3 \mathrm{mg} / \mathrm{L}$ ), and abnormal liver function tests (alanine and aspartate transaminases, alkaline phosphatase and gammaglutamyl transpeptidase were all elevated). A chest radiography revealed a bilateral abscessed pneumonia with right pleural effusion. Blood cultures were immediately initiated but no pulmonary sample was taken prior to antibiotherapy at the hospital. Treatment was started with ceftriaxone-spiramycin on the same day. The next day, blood culture was positive with S. aureus. As the strain was susceptible to meticillin and positive for Panton-Valentine leucocidin $(\mathrm{PVL})$ by polymerase chain reaction (PCR), treatment was changed to intravenous oxacillin and clindamycin per os. After initiation of this treatment, fever disappeared in only one day while haemoptysis persisted for seven days. No other pathogen was identified. After 21 days, treatment was stopped due to a drug rash with eosinophilia and systemic symptoms (DRESS) syndrome and replaced by linezolid up to a total duration of five weeks. S. aureus decolonisation was performed during hospitalisation to eradicate nasal carriage and to prevent recurrences.

\section{Case 2}

In November 2014, an 18-month-old child living in Mayotte presented at a dispensary with a three-day history of fever and right lower limb pain. They were referred to the hospital Emergency Department of Mamoudzou. At the time of admission, the clinical examination led to suspect arthritis of the right knee with a skin lesion in front and inflammatory extension to the rest of the leg. Body temperature was $39.8^{\circ} \mathrm{C}$ 
and heart rate $193 / \mathrm{min}$. Laboratory findings showed a white blood cell count of $13.4 \times 10^{9} / \mathrm{L}$ and a CRP of 229 $\mathrm{mg} / \mathrm{L}$. Ultrasound revealed joint effusion which could not be punctured. Blood cultures were immediately collected and treatment started with amoxicillin-clavulanic acid and gentamicin intravenously. The next day, the child was transferred to the intensive care unit due to worsening tachycardia and cold extremities despite the infusion of a volume expander. Blood culture was positive with $S$. aureus susceptible to meticillin. Based on suspicion of a toxin-mediated infection, treatment was changed to clindamycin, gentamicin, linezolid and polyvalent immunoglobulins intravenously. Blood culture became negative 48 hours after the initiation of antibiotherapy. Three days after admission, ultrasound showed joint effusion of the right knee associated with a collection at the quadriceps bursa. Culture from pus aspiration yielded an $S$. aureus isolate susceptible to meticillin. The $S$. aureus isolate was positive for PVL by PCR. Treatment was continued intravenously with oxacillin and clindamycin. Multiple pulmonary abscesses appeared on the tenth day, which led to change treatment for clindamycin-rifampicin and finally for amoxicillin-clavulanic acid-rifampicin orally for a total of six weeks, allowing the patient's recovery.

\section{Analysis of strains}

Blood cultures from the two patients yielded $S$. aureus isolates, which were sent to the French national reference centre for staphylococci in Lyon for characterisation.

The two strains were identified by matrix-assisted laser desorption/ionization time-of-flight (Maldi-Tof) mass spectrometry as S. aureus. Antimicrobial susceptibility testing was performed by disk diffusion assay or on automated Vitek2 system and interpreted according to the 2013 guidelines of the Antibiogram Committee of the French Society for Microbiology (CA-SFM). The following panel of antibiotics was tested: penicillin, oxacillin or cefoxitin, kanamycin, tobramycin, gentamicin, erythromycin, clindamycin or lincomycin, pristinamycin, levofloxacin or ofloxacin, tetracycline, rifampicin, trimethoprim-sulfamethoxazole, fusidic acid, fosfomycin, linezolid, teicoplanin, and vancomycin. The two isolates were susceptible to meticillin and to all the antibiotics tested except penicillin.

Further characterisation of the strains with the DNA microarray Identibac S. aureus Genotyping (Alere Technologies, Jena, Germany) for staphylococcal cassette chromosome (SCC) mec typing and toxin profiling [1] revealed that the two strains harboured the PVL genes and belonged to the $S$. argenteus lineage sequence type (ST) 2250/2277. This was confirmed by PVL PCR and by assignment by multilocus sequence typing (MLST) to ST2250 using specific primers for aroE [2]. Alignment of the seven MLST gene sequences from the patients' respective strains to those of $S$. argenteus, and neighbour-joining phylogenetic analysis showed that the two strains belonged to the $S$. argenteus lineage. Moreover, after 48 hours of growth, the two strains presented a lack of pigmentation typical of S. argenteus [3].

\section{Discussion}

This is, to the best of our knowledge, the first observation of $S$. argenteus lineage isolates harbouring the PVL genes. S. argenteus has recently been described as a novel species in the genus Staphylococcus, and is - as $S$. schweitzeri is - part of an $S$. aureus species complex [1]. Several studies previously described the $\mathrm{CC}_{75}$ lineage as grouping clones of $S$. aureus belonging to STs that are very distant from all other clones and characterised by the lack of staphyloxanthin (e.g. ST75, ST850, ST883, ST1223, ST1304, ST1850) [2-5]. Such strains were isolated mainly from indigenous populations in Australia and French Guyana $[2,4,6]$, but also in Cambodia, Fiji, Trinidad and Tobago, Thailand [7-10] and from animals in Africa [11,12]. No strain harbouring the PVL was described among these isolates, which were considered as having an attenuated virulence compared with other $S$. aureus strains $[5,13,14]$.

By confering cytotoxicity on neutrophils and monocytes-macrophages, PVL leads to a high degree of virulence [15]. PVL-positive strains are most frequently responsible for skin and soft-tissue infections, but can also cause severe necrotising pneumonia or aggressive bone and joint infections [16]. The present results indicate that some strains belonging to the $S$. argenteus lineage are able to acquire the PVL phage. Moreover, the severity of the clinical presentation of the two cases described in this report strongly suggests an important role of PVL in their infections.

Both cases described had a link to Mayotte which indicates the potential geographical origin of these isolates there. Enhanced surveillance is therefore necessary to determine whether PVL-positive S. argenteus lineage might emerge as a cause of infections in Mayotte and might disseminate further in France and in other countries.

\section{Conflict of interest}

None declared.

Authors' contributions

C. Dupieux was involved in clinical data collection with $R$. Blondé and S. Laurent, and in writing. C. Bouchiat, M. Bes, H. Meugnier and A. Tristan carried out the analysis of strains and revision of the drafts. F. Vandenesch, F. Laurent and A. Tristan critically revised the manuscript and carried out the final corrections. All authors read and approved the final manuscript.

References

1. Tong SYC, Schaumburg F, Ellington MJ, Corander J, Pichon $B$, Leendertz F, et al. Novel staphylococcal species that form part of a Staphylococcus aureus-related complex: the non-pigmented Staphylococcus argenteus sp. nov. and the 
non-human primate-associated Staphylococcus schweitzeri sp. nov. Int J Syst Evol Microbiol. 2015;65(Pt 1):15-22. http://dx.doi. org/10.1099/ijs.0.062752-o PMID:25269845

2. Ruimy R, Angebault C, Djossou F, Dupont C, Epelboin L, Jarraud $S$, et al. Are host genetics the predominant determinant of persistent nasal Staphylococcus aureus carriage in humans? J Infect Dis. 2010;202(6):924-34. http://dx.doi. org/10.1086/655901 PMID:20677941

3. Monecke S, Kanig H, Rudolph W, Müller E, Coombs G, Hotzel $\mathrm{H}$, et al. Characterisation of Australian MRSA strains ST75- and ST883-MRSA-IV and analysis of their accessory gene regulator locus. PLoS ONE. 2010;5(11):e14025. http://dx.doi.org/10.1371/ journal.pone.0014025 PMID:21103340

4. Ng JWS, Holt DC, Lilliebridge RA, Stephens AJ, Huygens F, Tong SYC, et al. Phylogenetically distinct Staphylococcus aureus lineage prevalent among indigenous communities in northern Australia. J Clin Microbiol. 2009;47(7):2295-300. http://dx.doi. org/10.1128/JCM.00122-09 PMID:19420161

5. Holt DC, Holden MTG, Tong SYC, Castillo-Ramirez S, Clarke L, Quail MA, et al. A very early-branching Staphylococcus aureus lineage lacking the carotenoid pigment staphyloxanthin. Genome Biol Evol. 2011;3(0):881-95. http://dx.doi.org/10.1093/ gbe/evro78 PMID:21813488

6. McDonald M, Dougall A, Holt D, Huygens F, Oppedisano F, Giffard PM, et al. Use of a single-nucleotide polymorphism genotyping system to demonstrate the unique epidemiology of methicillin-resistant Staphylococcus aureus in remote aboriginal communities. J Clin Microbiol. 2006;44(10):3720-7. http://dx.doi.org/10.1128/JCM.00836-06 PMID:17021102

7. Ruimy R, Armand-Lefevre L, Barbier F, Ruppé E, Cocojaru R, Mesli Y, et al. Comparisons between geographically diverse samples of carried Staphylococcus aureus. J Bacteriol. 2009;191(18):5577-83. http://dx.doi.org/10.1128/JB.00493-09 PMID:19592594〈/jrn〉

8. Jenney A, Holt D, Ritika R, Southwell P, Pravin S, Buadromo $\mathrm{E}$, et al. The clinical and molecular epidemiology of Staphylococcus aureus infections in Fiji. BMC Infect Dis. 2014;14(1):16o. http://dx.doi.org/10.1186/1471-2334-14-160 PMID:24655406

9. Monecke S, Stieber B, Roberts R, Akpaka PE, Slickers P, Ehricht R. Population structure of Staphylococcus aureus from Trinidad \& Tobago. PLoS ONE. 2014;9(2):e89120. http://dx.doi. org/10.1371/journal.pone.0089120 PMID:24586536

10. Thaipadungpanit J, Amornchai P, Nickerson EK, Wongsuvan G, Wuthiekanun V, Limmathurotsakul D, et al. Clinical and molecular epidemiology of Staphylococcus argenteus infections in Thailand. J Clin Microbiol. 2015;53(3):1005-8. http://dx.doi.org/10.1128/JCM.03049-14 PMID:25568440

11. Schaumburg F, Alabi AS, Köck R, Mellmann A, Kremsner PG, Boesch C, et al. Highly divergent Staphylococcus aureus isolates from African non-human primates. Environ Microbiol Rep. 2012;4(1):141-6. http://dx.doi.org/10.1111/j.17582229.2011.00316.x PMID:23757241

12. Akobi B, Aboderin $O$, Sasaki T, Shittu A. Characterization of Staphylococcus aureus isolates from faecal samples of the Straw-Coloured Fruit Bat (Eidolon helvum) in Obafemi Awolowo University (OAU), Nigeria. BMC Microbiol. 2012;12(1):279. http://dx.doi.org/10.1186/1471-2180-12-279 PMID:23181939

13. Tong SYC, Sharma-Kuinkel BK, Thaden JT, Whitney AR, Yang S-J, Mishra NN, et al. Virulence of endemic nonpigmented northern Australian Staphylococcus aureus clone (clonal complex 75, S. argenteus) is not augmented by staphyloxanthin. J Infect Dis. 2013;208(3):520-7. http://dx.doi. org/10.1093/infdis/jit173 PMID:23599317

14. Tong SYC, Lilliebridge RA, Bishop EJ, Cheng AC, Holt DC, McDonald MI, et al. Clinical correlates of Panton-Valentine leukocidin (PVL), PVL isoforms, and clonal complex in the Staphylococcus aureus population of Northern Australia. J Infect Dis. 2010;202(5):760-9. http://dx.doi. org/10.1086/655396 PMID:20662623

15. Boyle-Vavra S, Daum RS. Community-acquired methicillinresistant Staphylococcus aureus: the role of Panton-Valentine leukocidin. Lab Invest. 2007;87(1):3-9. http://dx.doi. org/10.1038/labinvest.3700501 PMID:17146447

16. Vandenesch F, Lina G, Henry T. Staphylococcus aureus hemolysins, bi-component leukocidins, and cytolytic peptides: a redundant arsenal of membrane-damaging virulence factors? Front Cell Infect Microbiol. 2012;2:12. http://dx.doi. org/10.3389/fcimb.2012.00012 PMID:22919604 